\title{
Multisensory integration mechanisms during aging
}

\author{
Jessica Freiherr ${ }^{1}{ }^{*}$, Johan N. Lundström ${ }^{2,3,4}$, Ute Habel ${ }^{5,6}$ and Kathrin Reetz ${ }^{6,7,8}$ \\ ${ }^{1}$ Diagnostic and Interventional Neuroradiology, RWTH Aachen University, Aachen, Germany \\ ${ }^{2}$ Department of Clinical Neuroscience, Karolinska Institute, Stockholm, Sweden \\ ${ }^{3}$ Monell Chemical Senses Center, Philadelphia, PA, USA \\ ${ }^{4}$ Department of Psychology, University of Pennsylvania, Philadelphia, PA, USA \\ ${ }^{5}$ Department of Psychiatry, Psychotherapy, and Psychosomatics, RWTH Aachen University, Aachen, Germany \\ 6 JARA BRAIN - Translational Brain Medicine, RWTH Aachen University, Aachen, Germany \\ ${ }^{7}$ Department of Neurology, RWTH Aachen University, Aachen, Germany \\ ${ }^{8}$ Institute of Neuroscience and Medicine (INM-4), Research Center Jülich, Jülich, Germany
}

\section{Edited by:}

Yu-Han Chen, University of New

Mexico, USA

\section{Reviewed by:}

Wen Li, University of

Wisconsin-Madison, USA

Jennifer L. Mozolic, Warren Wilson

College, USA

Jeannette R. Mahoney, Albert Einstein

College of Medicine, USA

*Correspondence:

Jessica Freiherr, Diagnostic and Interventional Neuroradiology, RWTH

Aachen University, Pauwelsstr. 30,

52074 Aachen, Germany

e-mail: jfreiherr@ukaachen.de
The rapid demographical shift occurring in our society implies that understanding of healthy aging and age-related diseases is one of our major future challenges. Sensory impairments have an enormous impact on our lives and are closely linked to cognitive functioning. Due to the inherent complexity of sensory perceptions, we are commonly presented with a complex multisensory stimulation and the brain integrates the information from the individual sensory channels into a unique and holistic percept. The cerebral processes involved are essential for our perception of sensory stimuli and becomes especially important during the perception of emotional content. Despite ongoing deterioration of the individual sensory systems during aging, there is evidence for an increase in, or maintenance of, multisensory integration processing in aging individuals. Within this comprehensive literature review on multisensory integration we aim to highlight basic mechanisms and potential compensatory strategies the human brain utilizes to help maintain multisensory integration capabilities during healthy aging to facilitate a broader understanding of age-related pathological conditions. Further our goal was to identify where further research is needed.

Keywords: crossmodal sensory integration, cognition, multimodal, aging neuroscience, elderly population multisensory integration

\section{MULTISENSORY INTEGRATION}

Each of our sensory systems provides us with a qualitatively distinct subjective complementary impression of our surrounding, which are of critical importance for perception, cognitive processing and control of action and can occur in a highly automatized manner (Meredith and Stein, 1983, 1985; Stein and Meredith, 1990). However, most of our everyday percepts are conveyed by multiple sensory systems like the olfactory, auditory, visual, gustatory, and tactile system. Our brain has the remarkable ability to integrate even disparate and complex multisensory information into a unique and coherent percept. The computational mechanisms responsible for this integration assure that the signal is amplified and any kind of accompanied noise is filtered out, thereby promoting the saliency of ecologically meaningful events. Input from two sensory channels, in comparison to a single one, increases the likelihood and speed of detecting and correctly identifying events (Gottfried and Dolan, 2003; Dematte et al., 2006, 2009) and also enhances sensory sensitivity (Dalton et al., 2000; Chen and Spence, 2011). It has also been indicated that higher cognitive sensory processing, like pleasantness evaluation of an odor, is enhanced when it is combined with a congruent auditory stimulus (Seo and Hummel, 2011). Overall, numerous results indicate that multisensory integration plays an important role in our daily life by facilitating and improving our perceptual capacities.

In more detail, multisensory integration is governed by four different principles. First, unimodal sensory stimuli need to be applied within a certain temporal sequence and second, spatial concordance is necessary in order to achieve optimal integration results (King and Palmer, 1985; Meredith and Stein, 1986). Thus, sensory stimuli of different modalities have to coincide with regards to time and space in order to be integrated. Third, contextual, semantic congruency, or correspondence, is fundamental for efficient multisensory integration (Spence, 2011). When those preconditions are fulfilled, the sensory stimuli appear as if emanating from the same object. Importantly, multisensory integration processes follow the principle of inverse effectivenesscross- or multisensory integration is most effective and therefore elicit maximal behavioral enhancements when less intense or weak and ambiguous individual stimuli are applied (Stein and Stanford, 2008).

Initial research on multisensory integration observed the superior colliculus of anesthetized cats as an important part of the neural network involved (Meredith and Stein, 1983, 1985; Stein and Meredith, 1990). Nowadays, it has been demonstrated that the traditional cortical network supporting multisensory integration 
in humans consists primarily of the superior temporal sulcus (STS) and the intraparietal sulcus (IPS); it has been suggested that the STS is associated with the integration and labeling of object identity, whereas the IPS is involved in a low-level spatial information processing (Calvert, 2001; Stein and Stanford, 2008). Further, the insula is considered a key area for detection of crossmodal coincidence and matching (Calvert, 2001). However, orbital and ventral areas of the frontal cortex and hippocampal areas have also been identified as neuronal correlates of bimodal multisensory integration involving the olfactory and gustatory domains (Gottfried and Dolan, 2003; Small and Prescott, 2005). Those areas might be responsible for attention and memory processing and are involved in novelty detection, congruency assessment, or task difficulty evaluation (Calvert, 2001). Within frontal areas, a dissociation with regard to object identity exists: the orbital network seems to be especially important for the integration of multisensory input related to food items while the ventrolateral prefrontal region mediates the assessment of non-food objects (Price, 2008).

The existing functional imaging research regarding multisensory integration processes can be divided into two topics: multisensory integration of (i) object observation and (ii) emotional perception. For the investigation of multisensory processes during object recognition, combinations of two different modalities - combined visual-auditory stimulations - is the most utilized. A concurrent combination of three different and congruent sensory stimuli has yet to be applied within a neuroimaging setting. However, when analyzing areas of overlap between the three senses of touch, sound, and vision, both the STS and IPS demonstrate a considerable overlap in processing (Bremmer et al., 2001; Beauchamp et al., 2008; Langner et al., 2012). In addition, the left fusiform gyrus (FG) seems to play a putative role within trimodal sensory object manipulation (Kassuba et al., 2011). Most of these studies are, however, based upon a combination of simple stimuli or a conjunction analysis of brain activation related to unimodal sensory stimulation. Multisensory integration mechanisms are of special importance during the perception and processing of emotions. Research from our own and other groups provides evidence for a neural network involving the amygdala, insula, frontal areas, FG, and STS which are responsible for integration of cross- or multisensory information related to emotional perception during stimulation with dynamic stimulus material of different modalities (Ethofer et al., 2006; Kreifelts et al., 2009; Seubert et al., 2010a,b; Klasen et al., 2011, 2012; Muller et al., 2011, 2012; Regenbogen et al., 2012a,b). Although we gained novel insights into multisensory integration processes with regards to object and emotion perception during the last years, a systematic investigation of multisensory integration in relation to differences between age groups and age-related pathologies using functional imaging means is still missing. Since emotional perception accounts for a major part of our everyday wellbeing and multisensory processes are heavily involved in emotional processes we want to draw attention towards a better understanding of multisensory processes and the underlying neural connections during emotional perception. Therefore, the aim of this review is to outline the knowledge about multisensory integration across the lifespan with a special focus on behavioral and neural correlates of multisensory integration during aging and age-related diseases. We further aim to identify areas where further research is needed in order to shed light onto the mechanisms of multisensory integration.

\section{MULTISENSORY INTEGRATION DURING AGING}

In light of the evolving demographic changes of our society, one important future task for the research communities is to further our understanding of lifelong healthy aging. Aging is a multifactorial and multidimensional process involving physiological, psychological, and social alterations. As part of ongoing degeneration throughout life, there is a progressive deterioration of physical function leading to loss of viability and an increase in vulnerability. Particularly, sensory impairments have an enormous impact on our lives and are closely related to intellectual functioning. Because we experience our environment through multiple sensory systems, which ultimately ensure our everyday safety, quality of life, and social adjustment, it is of interest to understand how multisensory integration processing changes as a function of healthy aging. In facing these challenges, the following question is noteworthy: what age-dependent changes in the neuronal integration of multisensory stimuli occurs in individuals experiencing healthy aging?

Effectiveness of multisensory integration depends upon functioning of the peripheral sensory organs as well as higher cognitive processes. As we age, we experience a decline of function in all our five senses-e.g., visual acuity decreases (Spear, 1993) and auditory thresholds increase (Liu and Yan, 2007). Olfactory capabilities are known to decline during aging as well (Rawson, 2006), however this deterioration can be attributed to a poor medical status and cognitive decline in the elderly (Nordin et al., 2012). Typically, motor speed and executive functions (Falkenstein et al., 2006), as well as working memory and attention control deteriorate as well (Fabiani, 2012). Previous structural neuroimaging studies, including voxel-based morphometry (VBM), deformation field morphometry (DBM), cortical thickness analyses, manual tracing techniques, and diffusion-weighted magnetic resonance imaging (MRI) have given some insights into the complexity of age-related structural brain changes (Sowell et al., 2004; Raz and Rodrigue, 2006; Sala et al., 2012). In a recent review, Hedman et al. (2012) concluded that apart from brain volume increases during childhood and adolescence, a continuous volume decrease of $0.2 \%$ per year can be observed, which accelerates to an annual brain volume loss of $0.5 \%$ at age 60 and more than $0.5 \%$ above the age of 60 years. Thereby, evidence is provided for a volume loss of gray matter and cortical thinning during aging. However, not only structural changes occur during lifespan. Evidence exists that older participants exhibit altered patterns of functional activation during cognitive tasks. The elderly engage brain areas (especially frontal areas) to a greater extent than young adults; this is most likely to compensate for impaired function in other brain areas (PosteriorAnterior Shift with Aging, PASA; Grady et al., 1994; Davis et al., 2008; Grady, 2012). In contrast to task-based methods, the taskindependent approach of a resting-state analysis is appealing due to its ability to assess altered brain function independent of the participant's active involvement and task understanding as well as independent of their sensory performance. The resting-state 
approach assesses altered brain functions caused by the summation of subtle physiological and pathological changes across the lifespan, which, in turn, can be linked to respective structural changes as noted above (Reetz et al., 2012). Given the extensive changes of perceptual and cognitive processes and the underlying structural and functional brain changes during healthy aging, it seems reasonable that multisensory integration performance as well is altered throughout the lifespan.

Research on multisensory integration in aging in relation to young adults mainly focused on visual-auditory paradigms and employed mostly static stimuli. These early studies indicated that older adults, when compared to younger adults, do not benefit from multisensory conditions (Stine et al., 1990; Walden et al., 1993; Sommers et al., 2005) or even report a suppressed cortical multisensory integration response in the elderly (Stephen et al., 2010). More recent studies, however, point towards an enhancement of multisensory integration effects also in older adults. Among others, shorter reaction times in response to multisensory events have been reported (Helfer, 1998; Laurienti et al., 2006; Diederich et al., 2008; Mahoney et al., 2011, 2012; Diaconescu et al., 2013). A potential reason for the earlier negative results and more recent positive results can be that the early studies focused on very complex auditory-visual speech perception and thus involve sensory as well as higher-order cognitive processes whereas later studies mostly used simpler combinations of stimuli originating from objects. Another explanation for the conflicting results is the use of different multisensory testing and data analysis techniques. It is also possible that the basic principles for a successful multisensory integration (temporal and spatial accordance, inverse effectiveness, semantic congruency) have been violated within the earlier studies.

Different aspects were discussed as basis for this improvement in multisensory integrative function in elder adults (Mozolic et al., 2012). General sensorimotor and cognitive slowing during aging is obviously not able to explain response times acceleration (Laurienti et al., 2006; Peiffer et al., 2007). However, it was recently demonstrated that older adults have a broader time window of integration as a consequence of increased response times and a wider distribution or range of response times; the combined effect aids older adults to separate stimuli in time (Diederich et al., 2008). In this study, older adults demonstrated a lower probability of integration due to the broader time window; however, in case of a successful integration the gain of older adults was larger compared to younger adults. Age-dependent deficits in top-down selective attention to incoming sensory information do not provide an explanation for the enhancement of multisensory integration in older subjects (Mozolic et al., 2008; Hugenschmidt et al., 2009). One plausible explanation is the principle of inverse effectiveness, i.e., that reduced sensitivity in the individual sensory systems (e.g., rigidity of the lenses, loss of hair cells in the ear and olfactory receptors) combined with age-related alterations in cognitive processing increases the relative magnitude of multisensory enhancements (Hairston et al., 2003). Thereby, multisensory integration becomes more important during aging as it helps to counteract the often-destructive consequences of unisensory deterioration. Mozolic et al. (2012) recently proposed a second possible explanation. They suggested that elderly do not adequately filter sensory noise and hence are more prone to distraction than their younger counterparts. As soon as the extraneous sensory information becomes relevant, however, older adults benefit from this enhanced processing of sensory background information. Evidence for a higher level of background sensory processing in the elderly was also provided by several resting-state studies pointing towards an increased default mode network (DMN) activity (Grady et al., 2006; Li et al., 2007). Furthermore, in elderly, the observed decrease in visual memory and visuo-constructive functions seems to be strongly associated with an age-dependent increase of functional connectivity specifically in the temporal lobe (Schlee et al., 2012).

Age-related changes during information processing (compensatory reallocation, neural compensation, dedifferentiation, inhibition) are based upon basic circuitry of the sensory systems involving several interactive neuronal loops such as prefronto-thalamo-cortical gating between the thalamus and the neocortex in order to effectively process sensory and higher-order cognitive information (Mahoney et al., 2011). Using magnetoencephalography, Diaconescu et al. (2013) indicated that sensory-specific regions showed an increased activity after visual-auditory stimulation in young and old participants, whereas inferior parietal and medial prefrontal areas responded preferentially in older subjects. Further, activation of the latter areas was related to faster detection of multisensory stimuli. This relation was mediated by age-related reductions in gray matter volume in those regions (Diaconescu et al., 2013). The authors propose that posterior parietal and medial prefrontal activity is the basis for the integrated response in older adults. This hypothesis is supported by the theory of PASA described above as well as the theory of cortical dedifferentiation stating that healthy aging is accompanied by decreased specificity of neurons in the prefrontal cortex (Park and Reuter-Lorenz, 2009).

Thus, the neural network governing multisensory integration displays clear age-dependent alterations and age-related changes in cognitive function have clear implications for multisensory processing. That said, although age is the number one major risk factor for a large range of degenerative diseases, it remains to be determined how multisensory integration is affected in different states of age-related diseases, and in particular, diseases of neurodegenerative nature. Unfortunately, to date, there are few published studies on this topic. Any potential changes due to neurodegenerative states might, however, be highly relevant as most of the age-related neurodegenerative disorders are preceded by long presymptomatic periods. Neuropathobiological changes occur many years, even decades, before the clinical manifestation of the disease and numerous compensatory mechanisms and neuroplastic capacities of the human brain remain to be clarified.

The most frequent age-related neurodegenerative disorder is Alzheimer's disease (AD). Due to aging of populations in both developed and developing societies, AD affects 24.3 million people worldwide and has become one of the most severe socioeconomical and medical burdens (Ferri et al., 2005). AD, the most common form of dementia, is a complex disease characterized by an accumulation of $\beta$-amyloid plaques and neurofibrillary tangles composed of tau amyloid fibrils. These changes are associated with synapse loss and neurodegeneration leading to a general and 
progressive loss of cognitive functions, initially predominantly manifested as memory impairment. As studies have revealed that increased DMN activity in healthy aging is associated with a higher level of background sensory processing (Grady et al., 2006; Li et al., 2007), functional changes in AD are of particular interest. Recent studies have mainly demonstrated task-induced deactivations of the DMN as well as a decreased DMN functional connectivity along a continuum from normal aging to pathological conditions (Andrews-Hanna et al., 2007; Hafkemeijer et al., 2012). Resting state functional MRI demonstrated that the regional coherence of brain activity is significantly altered in patients with AD [e.g., Filippi and Agosta, 2011]. Furthermore, abnormalities in the precuneus of patients with amnestic mild cognitive impairment compared to controls were found while $\mathrm{AD}$ patients showed alterations of large-scale functional brain networks extending well beyond the DMN. Moreover, episodicmemory related task-based functional neuroimaging studies in $\mathrm{AD}$ have revealed increased activity in the precuneus (Browndyke et al., 2013); an area which plays a crucial role in the DMN and has the highest metabolic and blood perfusion rates during resting conditions (Gusnard and Raichle, 2001). The clinical relevance of resting state networks beyond the DMN is notable because the degree of connectivity in the resting state networks may predict individual cognitive and emotional functions (Wang et al., 2010a). Specifically, interhemispheric functional connectivity of the hippocampi (Wang et al., 2010b), as well as connectivity between the hippocampus and the posteriomedial cortices, predicts memory performance in healthy individuals (Wang et al., 2010a). The available research demonstrates that suppression of the DMN during task performance is important. However, conceptualizing the network in terms of suppression implies that the DMN is a sort of nuisance network where its importance to voluntary cognitive functions lies primarily in minimizing its activity during tasks. Consequently, patients with $\mathrm{AD}$ that demonstrate hypometabolism in the precuneus/posterior cingulate component of the DMN (Bradley et al., 2002; Chetelat et al., 2008; Langbaum et al., 2009; Petrie et al., 2009; Schroeter et al., 2009) should have very good cognitive functioning during purposeful tasks because the DMN is not active. However, this is clearly not the case. Therefore, further studies with a focus on functional connectivity analyses might foster our understanding of changes in the context of multisensory integration in healthy populations and pathological conditions.

Overall, very little is known about behavioral benefits during multisensory integration in patients with age-related neurodegenerative disorders. Using bimodal stimulation (audio-visual speech presentation), patients demonstrate a limited ability to benefit from concurrent perceptual and linguistic cues compared to healthy aged subjects (Phillips et al., 2009). Given the fact that we have a poor understanding of multisensory integration in neurodegenerative disorders, further studies regarding behavioral and neuronal substrates of multisensory integration are warranted. The knowledge of mechanisms involved in multisensory integration in neurodegenerative disorders, with $\mathrm{AD}$ representing the most common one, is of high value given the fact that multisensory stimulation has an inherently high potential for early intervention and thus, also therapeutic application (Staal et al., 2003).

\section{CONCLUSION}

Advances are being made in disentangling the multisensory integration mechanisms in elderly, thus encouraging future larger longitudinal studies needed to understand the specific neurobiological and neuropathological basis of multisensory integration in health and disease. The current state of research on multisensory integration in healthy aging and age-related neurodegenerative disorders would greatly benefit from further studies as we aim to understand basic mechanisms and potential compensatory strategies of the human brain that help maintain multisensory integration capabilities during both healthy and pathological aging. Early identification of changes in multisensory integration will help to better inform choice of therapy and aid a personalized approach to clinical treatment. Future studies are warranted to determine the clinical translational value of multisensory integration processes in the elderly.

\section{ACKNOWLEDGMENTS}

Jessica Freiherr is supported by the Medical Faculty of RWTH Aachen University (IZKF-Interdisciplinary Centre for Clinical Research, START program). Johan N. Lundström is funded by the Knut and Alice Wallenberg Foundation (KAW 2012.0141) and the Swedish Research Council (VR 2009:3356). Kathrin Reetz is funded by the excellence Initiative of the German Research Foundation (DFG ZUK32/1).

\section{REFERENCES}

Andrews-Hanna, J. R., Snyder, A. Z., Vincent, J. L., Lustig, C., Head, D., Raichle, M. E., et al. (2007). Disruption of large-scale brain systems in advanced aging. Neuron 56, 924-935. doi: 10.1016/j.neuron.2007.10.038

Beauchamp, M. S., Yasar, N. E., Frye, R. E., and Ro, T. (2008). Touch, sound and vision in human superior temporal sulcus. Neuroimage 41, 1011-1020. doi: 10. 1016/j.neuroimage.2008.03.015

Bradley, K. M., O’sullivan, V. T., Soper, N. D., Nagy, Z., King, E. M., Smith, A. D., et al. (2002). Cerebral perfusion SPET correlated with Braak pathological stage in Alzheimer's disease. Brain 125, 1772-1781. doi: 10.1093/brain/awf185

Bremmer, F., Schlack, A., Shah, N. J., Zafiris, O., Kubischik, M., Hoffmann, K., et al. (2001). Polymodal motion processing in posterior parietal and premotor cortex: a human fMRI study strongly implies equivalencies between humans and monkeys. Neuron 29, 287-296. doi: 10.1016/s0896-6273(01)00198-2

Browndyke, J. N., Giovanello, K., Petrella, J., Hayden, K., Chiba-Falek, O., Tucker, K. A., et al. (2013). Phenotypic regional functional imaging patterns during memory encoding in mild cognitive impairment and Alzheimer's disease. Alzheimers Dement. 9, 284-294. doi: 10.1016/j.jalz.2011.12.006

Calvert, G. A. (2001). Crossmodal processing in the human brain: insights from functional neuroimaging studies. Cereb. Cortex 11, 1110-1123. doi: 10 . 1093/cercor/11.12.1110

Chen, Y. C., and Spence, C. (2011). Crossmodal semantic priming by naturalistic sounds and spoken words enhances visual sensitivity. J. Exp. Psychol. Hum. Percept. Perform. 37, 1554-1568. doi: 10.1037/a0024329

Chetelat, G., Desgranges, B., Landeau, B., Mezenge, F., Poline, J. B., De La Sayette, V., et al. (2008). Direct voxel-based comparison between grey matter hypometabolism and atrophy in Alzheimer's disease. Brain 131, 60-71. doi: 10. 1093/brain/awm288

Dalton, P., Doolittle, N., Nagata, H., and Breslin, P. A. (2000). The merging of the senses: integration of subthreshold taste and smell. Nat. Neurosci. 3, 431-432. doi: $10.1038 / 74797$

Davis, S. W., Dennis, N. A., Daselaar, S. M., Fleck, M. S., and Cabeza, R. (2008). Que PASA? The posterior-anterior shift in aging. Cereb. Cortex 18, 1201-1209. doi: 10.1093/cercor/bhm155

Dematte, L. M., Sanabria, D., and Spence, C. (2006). Cross-modal associations between odors and colors. Chem. Senses 31, 531-538. doi: 10 1093/chemse/bjj057 
Dematte, M. L., Sanabria, D., and Spence, C. (2009). Olfactory discrimination: when vision matters? Chem. Senses 34, 103-109. doi: 10.1093/chemse/bjn 055

Diaconescu, A. O., Hasher, L., and McIntosh, R. A. (2013). Visual dominance and multisensory integration changes with age. Neuroimage 65, 152-166. doi: 10. 1016/j.neuroimage.2012.09.057

Diederich, A., Colonius, H., and Schomburg, A. (2008). Assessing age-related multisensory enhancement with the time-window-of-integration model. Neuropsychologia 46, 2556-2562. doi: 10.1016/j.neuropsychologia.2008.03. 026

Ethofer, T., Pourtois, G., and Wildgruber, D. (2006). Investigating audiovisual integration of emotional signals in the human brain. Prog. Brain Res. 156, 345361. doi: 10.1016/s0079-6123(06)56019-4

Fabiani, M. (2012). It was the best of times, it was the worst of times: a psychophysiologist's view of cognitive aging. Psychophysiology 49, 283-304. doi: 10.1111/j. 1469-8986.2011.01331.x

Falkenstein, M., Yordanova, J., and Kolev, V. (2006). Effects of aging on slowing of motor-response generation. Int. J. Psychophysiol. 59, 22-29. doi: 10.1016/j. ijpsycho.2005.08.004

Ferri, C. P., Prince, M., Brayne, C., Brodaty, H., Fratiglioni, L., Ganguli, M., et al. (2005). Global prevalence of dementia: a Delphi consensus study. Lancet 366, 2112-2117. doi: 10.1016/s0140-6736(05)67889-0

Filippi, M., and Agosta, F. (2011). Structural and functional network connectivity breakdown in Alzheimer's disease studied with magnetic resonance imaging techniques. J. Alzheimers Dis. 24, 455-474. doi: 10.3233/JAD-2011-101854

Gottfried, J. A., and Dolan, R. J. (2003). The nose smells what the eye sees: crossmodal visual facilitation of human olfactory perception. Neuron 39, 375386. doi: 10.1016/s0896-6273(03)00392-1

Grady, C. (2012). The cognitive neuroscience of ageing. Nat. Rev. Neurosci. 13, 491505. doi: 10.1038/nrn3256

Grady, C. L., Maisog, J. M., Horwitz, B., Ungerleider, L. G., Mentis, M. J., Salerno, J. A., et al. (1994). Age-related changes in cortical blood flow activation during visual processing of faces and location. J. Neurosci. 14, 1450-1462.

Grady, C. L., Springer, M. V., Hongwanishkul, D., Mcintosh, A. R., and Winocur, G. (2006). Age-related changes in brain activity across the adult lifespan. J. Cogn. Neurosci. 18, 227-241. doi: 10.1162/jocn.2006.18.2.227

Gusnard, D. A., and Raichle, M. E. (2001). Searching for a baseline: functional imaging and the resting human brain. Nat. Rev. Neurosci. 2, 685-694. doi: 10. $1038 / 35094500$

Hafkemeijer, A., Van Der Grond, J., and Rombouts, S. A. (2012). Imaging the default mode network in aging and dementia. Biochim. Biophys. Acta 1822, 431441. doi: 10.1016/j.bbadis.2011.07.008

Hairston, W. D., Laurienti, P. J., Mishra, G., Burdette, J. H., and Wallace, M. T. (2003). Multisensory enhancement of localization under conditions of induced myopia. Exp. Brain Res. 152, 404-408. doi: 10.1007/s00221-003-1646-7

Hedman, A. M., Van Haren, N. E., Schnack, H. G., Kahn, R. S., and Hulshoff Pol, H. E. (2012). Human brain changes across the life span: a review of 56 longitudinal magnetic resonance imaging studies. Hum. Brain Mapp. 33, 19872002. doi: $10.1002 / \mathrm{hbm} .21334$

Helfer, K. S. (1998). Auditory and auditory-visual recognition of clear and converational speech by older adults. J. Am. Acad. Audiol. 9, 234-242.

Hugenschmidt, C. E., Mozolic, J. L., and Laurienti, P. J. (2009). Suppression of multisensory integration by modality-specific attention in aging. Neuroreport 20, 349-353. doi: 10.1097/wnr.0b013e328323ab07

Kassuba, T., Klinge, C., Holig, C., Menz, M. M., Ptito, M., Roder, B., et al. (2011). The left fusiform gyrus hosts trisensory representations of manipulable objects. Neuroimage 56, 1566-1577. doi: 10.1016/j.neuroimage.2011.02.032

King, A. J., and Palmer, A. R. (1985). Integration of visual and auditory information in bimodal neurones in the guinea-pig superior colliculus. Exp. Brain Res. 60, 492-500. doi: 10.1007/bf00236934

Klasen, M., Chen, Y. H., and Mathiak, K. (2012). Multisensory emotions: perception, combination and underlying neural processes. Rev. Neurosci. 23, 381-392. doi: 10.1515/revneuro-2012-0040

Klasen, M., Kenworthy, C. A., Mathiak, K. A., Kircher, T. T., and Mathiak, K. (2011). Supramodal representation of emotions. J. Neurosci. 31, 13635-13643. doi: 10. 1523/jneurosci.2833-11.2011

Kreifelts, B., Ethofer, T., Shiozawa, T., Grodd, W., and Wildgruber, D. (2009). Cerebral representation of non-verbal emotional perception: fMRI reveals audiovisual integration area between voice- and face-sensitive regions in the superior temporal sulcus. Neuropsychologia 47, 3059-3066. doi: 10.1016/j. neuropsychologia.2009.07.001

Langbaum, J. B., Chen, K., Lee, W., Reschke, C., Bandy, D., Fleisher, A. S., et al. (2009). Categorical and correlational analyses of baseline fluorodeoxyglucose positron emission tomography images from the Alzheimer's Disease Neuroimaging Initiative (ADNI). Neuroimage 45, 1107-1116. doi: 10.1016/j. neuroimage.2008.12.072

Langner, R., Kellermann, T., Eickhoff, S. B., Boers, F., Chatterjee, A., Willmes, K., et al. (2012). Staying responsive to the world: modality-specific and-nonspecific contributions to speeded auditory, tactile and visual stimulus detection. Hum. Brain Mapp. 33, 398-418. doi: 10.1002/hbm.21220

Laurienti, P. J., Burdette, J. H., Maldjian, J. A., and Wallace, M. T. (2006). Enhanced multisensory integration in older adults. Neurobiol. Aging 27, 11551163. doi: 10.1016/j.neurobiolaging.2005.05.024

Li, C. S., Yan, P., Bergquist, K. L., and Sinha, R. (2007). Greater activation of the "default" brain regions predicts stop signal errors. Neuroimage 38, 640-648. doi: 10.1016/j.neuroimage.2007.07.021

Liu, X. Z., and Yan, D. (2007). Ageing and hearing loss. J. Pathol. 211, 188-197. doi: $10.1002 /$ path. 2102

Mahoney, J. R., Li, P. C. C., Oh-Park, M., Verghese, J., and Holtzer, R. (2011). Multisensory integration across the senses in young and old adults. Brain Res. 1426, 43-53. doi: 10.1016/j.brainres.2011.09.017

Mahoney, J. R., Verghese, J., Dumas, K., Wang, C., and Holtzer, R. (2012). The effect of multisensory cues on attention in aging. Brain Res. 1472, 63-73. doi: 10. 1016/j.brainres.2012.07.014

Meredith, M. A., and Stein, B. E. (1983). Interactions among converging sensory inputs in the superior colliculus. Science 221, 389-391. doi: 10.1126/science. 6867718

Meredith, M. A., and Stein, B. E. (1985). Descending efferents form the superior colliculus relay integrated multisensory infromation. Science 227, 657-659. doi: 10.1126/science. 3969558

Meredith, M. A., and Stein, B. E. (1986). Visual, auditory and somatosensory convergence on cells in superior colliculus results in multisensory integration. J. Neurophysiol. 56, 640-662.

Mozolic, J. L., Hugenschmidt, C. E., Peiffer, A. M., and Laurienti, P. J. (2008). Modality-specific selective attention attenuates multisensory integration. Exp. Brain Res. 184, 39-52. doi: 10.1007/s00221-007-1080-3

Mozolic, J. L., Hugenschmidt, C. E., Pfeiffer, A. M., and Laurienti, P. J. (2012). "Multisensory integration and aging," in The Neural Bases of Multisensory Processes, eds M. M. Murray and M. T. Wallace (Boca Raton, FL: CRC Press). http://www.ncbi.nlm.nih.gov/books/NBK92841/

Muller, V. I., Cieslik, E. C., Turetsky, B. I., and Eickhoff, S. B. (2012). Crossmodal interactions in audiovisual emotion processing. Neuroimage 60, 553-561. doi: 10.1016/j.neuroimage.2011.12.007

Muller, V. I., Habel, U., Derntl, B., Schneider, F., Zilles, K., Turetsky, B. I., et al. (2011). Incongruence effects in crossmodal emotional integration. Neuroimage 54, 2257-2266. doi: 10.1016/j.neuroimage.2010.10.047

Nordin, S., Almkvist, O., and Berglund, B. (2012). Is loss in odor sensitivity inevitable in the aging individual? A study of "successfully aged" elderly. Chem. Percept. 5, 188-196. doi: 10.1007/s12078-011-9102-8

Park, D. C., and Reuter-Lorenz, P. (2009). The adaptive brain: aging and neurocognitive scaffolding. Annu. Rev. Psychol. 60, 173-196. doi: 10.1146/annurev.psych. 59.103006.093656

Peiffer, A. M., Mozolic, J. L., Hugenschmidt, C. E., and Laurienti, P. J. (2007). Age-related multisensory enhancement in a simple audiovisual detection task. Neuroreport 18, 1077-1081. doi: 10.1097/wnr.0b013e3281e72ae7

Petrie, E. C., Cross, D. J., Galasko, D., Schellenberg, G. D., Raskind, M. A., Peskind, E. R., et al. (2009). Preclinical evidence of Alzheimer changes: convergent cerebrospinal fluid biomarker and fluorodeoxyglucose positron emission tomography findings. Arch. Neurol. 66, 632-637. doi: 10.1001/archneurol. 2009.59

Phillips, N. A., Baum, S., and Taler, V. (2009). "Audio-visual speech perception in mild cognitive impairment and healthy elderly controls", in AVSP 2009 International Conference on Audio-Visual Speech Processing. Norwich, UK.

Price, J. L. (2008). Multisensory convergence in the orbital and ventrolateral prefrontal cortex. Chemosens. Percept. 1, 103-109. doi: 10.1007/s12078-0089013-5

Rawson, N. E. (2006). Olfactory loss in aging. Sci. Aging Knowledge Environ. 2006:pe6. doi: 10.1126/sageke.2006.5.pe6 
Raz, N., and Rodrigue, K. M. (2006). Differential aging of the brain: patterns, cognitive correlates and modifiers. Neurosci. Biobehav. Rev. 30, 730-748. doi: 10. 1016/j.neubiorev.2006.07.001

Reetz, K., Nickl-Jockschat, T., Kleiman, A., Schulz, J. B., Scheider, F., Laird, A. R., et al. (2012). Neuroanatomic changes and their association with cognitive decline in miled cognitive impairment: a meta-analysis. Brain Struct. Funct. 217, 115-125. doi: 10.1007/s00429-011-0333-x

Regenbogen, C., Schneider, D. A., Finkelmeyer, A., Kohn, N., Derntl, B., Kellermann, T., et al. (2012a). The differential contribution of facial expressions, prosody and speech content to empathy. Cogn. Emot. 26, 995-1014. doi: 10. 1080/02699931.2011.631296

Regenbogen, C., Schneider, D. A., Gur, R. E., Schneider, F., Habel, U., and Kellermann, T. (2012b). Multimodal human communication - Targeting facial expressions, speech content and prosody. Neuroimage 60, 2346-2356. doi: 10. 1016/j.neuroimage.2012.02.043

Sala, S., Agosta, F., Pagani, E., Copetti, M., Comi, G., and Filippi, M. (2012). Microstructural changes and atrophy in brain white matter tracts with aging. Neurobiol. Aging 33, 488-498, e482. doi: 10.1016/j.neurobiolaging.2010. 04.027

Schlee, W., Leirer, V., Kolassa, S., Thurm, F., Elbert, T., and Kolassa, I. T. (2012). Development of large-scale functional networks over the lifespan. Neurobiol. Aging 33, 2411-2421. doi: 10.1016/j.neurobiolaging.2011.11.031

Schroeter, M. L., Stein, T., Maslowski, N., and Neumann, J. (2009). Neural correlates of Alzheimer's disease and mild cognitive impairment: a systematic and quantitative meta-analysis involving 1351 patients. Neuroimage 47, 1196-1206. doi: 10.1016/j.neuroimage.2009.05.037

Seo, H. S., and Hummel, T. (2011). Auditory-olfactory integration: congruent or pleasant sounds amplify odor pleasantness. Chem. Senses 36, 301-309. doi: 10. 1093/chemse/bjq129

Seubert, J., Kellermann, T., Loughead, J., Boers, F., Brensinger, C., Schneider, F., et al. (2010a). Processing of disgusted faces is facilitated by odor primes: a functional MRI study. Neuroimage 53, 746-756. doi: 10.1016/j.neuroimage. 2010.07.012

Seubert, J., Loughead, J., Kellermann, T., Boers, F., Brensinger, C. M., and Habel, U. (2010b). Multisensory integration of emotionally valenced olfactory-visual information in patients with schizophrenia and healthy controls. J. Psychiatry Neurosci. 35, 185-194. doi: 10.1503/jpn.090094

Small, D. M., and Prescott, J. (2005). Odor/taste integration and the perception of flavor. Exp. Brain Res. 166, 345-357. doi: 10.1007/s00221-005-2376-9

Sommers, M. S., Tye-Murray, N., and Spehar, B. (2005). Auditory-visual speech perception and auditory-visual enhancement in normal-hearing younger and older adults. Ear Hear. 26, 263-275. doi: 10.1097/00003446-20050600000003

Sowell, E. R., Thompson, P. M., and Toga, A. W. (2004). Mapping changes in the human cortex throughout the span of life. Neuroscientist 10, 372-392. doi: 10. $1177 / 1073858404263960$
Spear, P. D. (1993). Neural bases of visual deficits during aging. Vision Res. 33, 2589 2609. doi: 10.1016/0042-6989(93)90218-1

Spence, C. (2011). Crossmodal correspondences: a tutorial review. Atten. Percept. Psychophys. 73, 971-995. doi: 10.3758/s13414-010-0073-7

Staal, J., Pinkney, L., and Roane, D. (2003). Assessment of stimulus preference in Snoezelen therapy for elderly with dementia. Br. J. Occup. Ther. 66, 542-551.

Stein, B. E., and Meredith, M. A. (1990). Multisensory integration. Neural and behavioral solutions for dealing with stimuli from different sensory modalities. Ann. N Y Acad. Sci. 608, 51-65. doi: 10.1111/j.1749-6632.1990.tb48891.x

Stein, B. E., and Stanford, T. R. (2008). Multisensory integration: current issues from the perspective of the single neuron. Nat. Rev. Neurosci. 9, 255-266. doi: 10. 1038/nrn2331

Stephen, J. M., Knoefel, J. E., Adair, J., Hart, B., and Aine, C. J. (2010). Aging-related changes in auditory and visual integration measured with MEG. Neurosci. Lett. 484, 76-80. doi: 10.1016/j.neulet.2010.08.023

Stine, E. A., Wingfield, A., and Myers, S. D. (1990). Age differences in processing information from television news: the effects of bisensory augmentation. $J$. Gerontol. 45, P1-P8. doi: 10.1093/geronj/45.1.p1

Walden, B. E., Busacco, D. A., and Montgomery, A. A. (1993). Benefit from visual cues in auditory-visual speech recognition by middle-aged and elderly persons. J. Speech Hear. Res. 36, 431-436.

Wang, L., Laviolette, P., O’keefe, K., Putcha, D., Bakkour, A., Van Dijk, K. R., et al. (2010a). Intrinsic connectivity between the hippocampus and posteromedial cortex predicts memory performance in cognitively intact older individuals. Neuroimage 51, 910-917. doi: 10.1016/j.neuroimage.2010.02.046

Wang, L., Negreira, A., Laviolette, P., Bakkour, A., Sperling, R. A., and Dickerson, B. C. (2010b). Intrinsic interhemispheric hippocampal functional connectivity predicts individual differences in memory performance ability. Hippocampus 20, 345-351. doi: 10.1002/hipo.20771

Conflict of Interest Statement: The authors declare that the research was conducted in the absence of any commercial or financial relationships that could be construed as a potential conflict of interest.

Received: 12 June 2013; accepted: 26 November 2013; published online: 13 December 2013.

Citation: Freiherr J, Lundström JN, Habel U and Reetz K (2013) Multisensory integration mechanisms during aging. Front. Hum. Neurosci. 7:863. doi: 10.3389/fnhum.2013.00863

This article was submitted to the journal Frontiers in Human Neuroscience.

Copyright (C) 2013 Freiherr, Lundström, Habel and Reetz. This is an open-access article distributed under the terms of the Creative Commons Attribution License (CC $B Y)$. The use, distribution or reproduction in other forums is permitted, provided the original author(s) or licensor are credited and that the original publication in this journal is cited, in accordance with accepted academic practice. No use, distribution or reproduction is permitted which does not comply with these terms. 